\title{
Noether charges and black hole mechanics in Einstein-æether theory
}

\author{
Brendan Z. Fostert* \\ Dept. of Physics, University of Maryland, \\ College Park, MD 20742-4111, USA
}

(Dated: December 9, 2005)

\begin{abstract}
The Noether charge method for defining the Hamiltonian of a diffeomorphism-invariant field theory is applied to "Einstein-æther" theory, in which gravity couples to a dynamical, timelike, unit-norm vector field. Using the method, expressions are obtained for the total energy, momentum, and angular momentum of an Einstein-æether space-time. The method is also used to discuss the mechanics of Einstein-æther black holes. The derivation of Wald, and Iyer and Wald, of the first law of black hole thermodynamics fails for this theory, because the unit vector is necessarily singular at the bifurcation surface of the Killing horizon. A general identity relating variations of energy and angular momentum to a surface integral at the horizon is obtained, but a thermodynamic interpretation, including a definitive expression for the black hole entropy, is not found.

PACS numbers: 04.50.+h, 04.70.Bw
\end{abstract}

*bzf@umd.edu 


\section{INTRODUCTION}

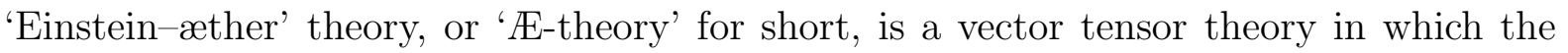
vector field, or 'æether', is constrained to be everywhere timelike and of fixed norm. This theory has received increasing attention lately, stemming from a broader interest in the possibility that Lorentz symmetry is not an exact symmetry of nature. The vector field defines a "preferred" frame, thus allowing for violations of local Lorentz symmetry, while its status as a dynamical field preserves diffeomorphism invariance. The condition on the vector norm (which can always be scaled to unity) ensures that the æether just picks out a timelike direction, and is required for stability of the theory at the semi-classical level [1]. For a review of properties of this theory and references to earlier work, see [2].

The conventional Æ-theory action, defined below, contains four free parameters. Constraints on the acceptable values of the parameters are implied by observational evidence, but one can also argue for limits imposed by theoretical considerations (for a summary of the known constraints, see [3]). A possible requirement that motivates the present work is that the theory should satisfy some form of energy positivity. It may be that imposing positivity for all solutions is more restrictive than necessary, or perhaps that one should only require positivity in the rest-frame of the æther. Whatever the argument, an expression for the energy is required to know how the parameters are constrained.

With this goal in mind, I give here an expression for the total energy of an asymptoticallyflat Æ-theory space-time, as well as expressions for the total momentum and angular momentum. These are generated via the 'Noether charge' method [4, 5] of defining the value of the on-shell Hamiltonian for a diffeomorphism-invariant field theory, directly from the theory's Lagrangian. The conventional ADM and Komar expressions, which have the form of integrals at spatial infinity, acquire æether-dependent corrections due to the non-vanishing of the æther at infinity. Parameter constraints are not discussed. The results here complement those of Eling [6], in which expressions for the total energy and the energy of linearized wave-modes are derived via pseudotensor methods.

The Noether-charge method also allows one to write down a differential identity that governs variations of stationary, axi-symmetric black hole solutions. As shown by Wald [4] and Iyer and Wald [5], in a wide variety of theories this identity can be massaged into the familiar form of the 'first law' of black hole mechanics and then interpreted as a law of 
thermodynamics. The recent discovery of $Æ$-theory black hole solutions [7] motivates the study of the first law for Æ-theory black holes. The authors of [7] demonstrate existence of these solutions, but have not found analytic expressions for the fields; therefore, the form of the first law cannot be inferred directly from the solutions. One can, however, attempt to derive the law via the Noether charge method. Unfortunately, the algorithms of [4, 5]

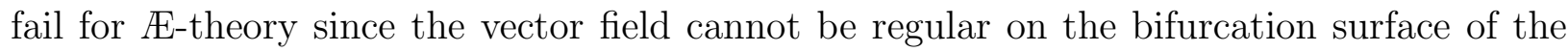
horizon, where a crucial calculation is performed. Below, a law resembling the first law is derived by less elegant means for static, spherically-symmetric solutions, but a thermodynamic interpretation of this expression is not given. In particular, a definitive expression for the horizon entropy in Æ-theory has not yet been found.

The Noether charge methodology is briefly reviewed in Section [I] The requisite differential forms for Æ-theory are derived in Section [II. These are used to determine expressions for the total energy, momentum, and angular momentum of an asymptotically-flat Æ-theory space-time in Section IV] The first law of Æ-theory black holes is discussed in Section V] I follow the conventions of [8], except that the metric here has signature (+---). I employ units in which $\hbar=c=1$.

\section{NOETHER CHARGE METHODOLOGY}

I will summarize here the application of the Noether charge method [4, $[5]$ to the definition of total energy, momentum, and angular momentum of an asymptotically-flat space-time. (We take the space-time to be four-dimensional, but the method can be applied in any dimension.) Given a diffeomorphism-invariant field theory defined from an action principle, one can construct a phase space with symplectic structure from the space of field configurations and the theory's Lagrangian. For the case of an Æ-theory system on a globally hyperbolic space-time, the phase space structure permits a well-defined Hamiltonian formulation. For every diffeomorphism on space-time, generated by vector field $\xi^{a}$, there is a corresponding evolution in phase space, with Hamiltonian generator $H_{\xi}$. This generator is implicitly defined through Hamilton's equation, which takes the form [4, 5]

$$
\delta H=\int_{C}\left(\delta \mathbf{J}-d\left(i_{\xi} \boldsymbol{\Theta}\right)\right)
$$


where $\mathbf{J}$ and $\boldsymbol{\Theta}$ are differential 3-forms that depend on the dynamical fields, a variation of the fields, and the vector field $\xi^{a}$; the surface of integration is a space-like Cauchy surface $C$ of the space-time.

The forms $\boldsymbol{\Theta}$ and $\mathbf{J}$ are obtained from the theory's Lagrangian as follows. Let the Lagrangian $\mathbf{L}$ be a four-form constructed locally out of the dynamical fields, denoted collectively by $\psi$. The 3 -form $\boldsymbol{\Theta}$ is defined by the variation of $\mathbf{L}$ due to a variation of $\psi$ :

$$
\delta \mathbf{L}=\mathbf{E}[\psi] \cdot \delta \psi+d \mathbf{\Theta}[\delta \psi]
$$

where $\mathbf{E}[\psi]$ are identified as the equations of motion for the fields, the dot representing contraction over appropriate indices.

This definition only determines $\Theta$ up to the addition of a closed form, which must be exact by the result of [9]. The contribution to $\delta H$ (10) from an asymptotic boundary is typically not effected by such ambiguity, though, since the fall-off conditions on the dynamical fields that guarantee convergence of $\delta H$ imply that any covariant, exact three-form added to $\Theta$ will give no asymptotic contribution to $\delta H$. Such is the case for $\mathbb{E}$-theory with the conditions chosen below. A contribution might arise given an inner boundary to the space-time. Here (Sec. V] we only consider stationary configurations on such space-times, and one can then show that the contribution to $\delta H$ vanishes. We will therefore fix the definition of $\Theta$ by taking the "most-obvious" choice that emerges from variation of the Lagrangian.

To each vector field on space-time $\xi^{a}$, we associate the Noether current 3 -form $\mathbf{J}[\xi]$,

$$
\mathbf{J}[\xi]=\boldsymbol{\Theta}\left[\mathcal{L}_{\xi} \psi\right]-i_{\xi} \mathbf{L}
$$

This current is conserved, $d \mathbf{J}=0$, for arbitrary $\xi^{a}$ when $\psi$ satisfies the equations of motion. This fact implies [9] that $\mathbf{J}$ can be expressed in the form

$$
\mathbf{J}[\xi]=d \mathbf{Q}[\xi]
$$

when $\mathbf{E}[\psi]=0$. If in addition $\delta \psi$ is such that the equations of motion linearized about $\psi$ are satisfied, then $\delta \mathbf{J}=d \delta \mathbf{Q}$, where here and below we choose $\delta \xi^{a}=0$. $\mathbf{Q}$ is only defined up to addition of a closed, hence exact [9], 2-form, but this ambiguity does not effect $\delta H$. We will therefore fix the definition of $\mathbf{Q}$ by taking the "most-obvious" choice.

An additional ambiguity can arise if one thinks of the Lagrangian $\mathbf{L}$ as defined only up to the addition of a boundary term, i.e. an exact 4-form. Adding such a form to $\mathbf{L}$ effects 
$\boldsymbol{\Theta}, \mathbf{J}$, and $\mathbf{Q}$ individually but leads to no net effect on $\delta H$. We will fix this ambiguity by again taking the "most-obvious" choices for the forms.

The Hamiltonian differential evaluated on-shell — when the full and linearized equations of motion are satisfied - is thus a surface term

$$
\delta H_{\xi}=\int_{\partial C}\left(\delta \mathbf{Q}-i_{\xi} \mathbf{\Theta}\right) .
$$

We will restrict attention to the case where $C$ is asymptotically flat at spatial infinity. The boundary of $C$ will consist of a surface "at infinity" - the limit of a two-sphere whose radius is taken to infinity - and a possible inner surface, such as a black hole horizon.

One can define a Hamiltonian function $H_{\xi}$ if there exists a 2-form $\mathbf{B}$ such that

$$
\int_{\partial C} \delta\left(i_{\xi} \mathbf{B}\right)=\int_{\partial C} i_{\xi} \boldsymbol{\Theta} .
$$

We then define the Hamiltonian as

$$
H_{\xi}=\int_{\partial C}\left(\mathbf{Q}-i_{\xi} \mathbf{B}\right) .
$$

We can assume that the fall-off conditions on the fields are such that at infinity, $d\left(\mathbf{Q}-i_{\xi} \mathbf{B}\right)=$ 0 . It follows that the value of the contribution to $H_{\xi}$ from the surface at infinity is conserved and can be interpreted as the conserved quantity associated with the symmetry generated by $\xi^{a}$.

One can define the total energy $\mathcal{E}$ of the space-time to be the value of the asymptotic Hamiltonian for the case where $\xi^{a}$ is a time translation $t^{a}=(\partial / \partial t)^{a}$ at infinity

$$
\mathcal{E}=\int_{\infty}\left(\mathbf{Q}[t]-i_{t} \mathbf{B}\right) .
$$

Likewise, with $x_{i}^{a}=\left(\partial / \partial x_{i}\right)^{a}$ a constant, spatial translation at infinity, one can define the total momentum in the $x_{i}^{a}$-direction $\mathcal{P}_{i}$ as

$$
\mathcal{P}_{i}=-\int_{\infty}\left(\mathbf{Q}\left[x_{i}\right]-i_{x_{i}} \mathbf{B}\right) .
$$

One can define the total angular momentum $\mathcal{J}$ (about a particular axis) via a vector field $\varphi^{a}$ that is a rotation at infinity, tangent to the bounding 2-sphere. The pull-back to the boundary of $i_{\varphi} \mathbf{B}$ vanishes, giving

$$
\mathcal{J}=-\int_{\infty} \mathbf{Q}[\varphi] .
$$


I note parenthetically that it follows from this definition that the total angular momentum must be zero for any axi-symmetric configuration (one for which $\mathcal{L}_{\varphi} \psi=0$ ), on $C$ possessing no inner boundary. This follows from the vanishing of $\mathbf{J}[\varphi]=d \mathbf{Q}[\varphi]$, when evaluated on such a configuration and pulled back to $C$. This result does not appear to have been stated explicitly with this generality before, although an early application is found in the proof of Cohen and Wald [10] that there are no rotating, axi-symmetric geons, in work that predates the precise formulation of the Noether charge method. This result also provides a short proof that there can be no rotating, axi-symmetric boson stars in general relativity. This generalizes the known result [11] that are no stationary, rotating, axi-symmetric boson stars. (Here, axi-symmetry must include any complex argument of the scalar field, as well as its modulus; this is a stronger sense of 'axi-symmetric' than is common in the boson-star-related literature.) In the presence of an inner boundary, such as an event horizon, the vanishing of $d \mathbf{Q}$ implies that the total angular momentum, i.e. the integral of $\mathbf{Q}$ over the boundary at infinity, is equal to the integral of $\mathbf{Q}$ over the inner boundary. Consequently, this result is not in conflict with the existence of rotating, axi-symmetric black holes.

\section{III. Æ-THEORY FORMS}

In this section, we will give the explicit expressions of the differential forms defined above, for Æ-theory. The conventional, second-order Æ-theory Lagrangian 4-form $\mathbf{L}$ is

$$
\begin{aligned}
\mathbf{L}=\frac{-1}{16 \pi G}(R & +c_{1}\left(\nabla_{a} u_{b}\right)\left(\nabla^{a} u^{b}\right)+c_{2}\left(\nabla_{a} u^{a}\right)\left(\nabla_{b} u^{b}\right) \\
& \left.+c_{3}\left(\nabla_{a} u^{b}\right)\left(\nabla_{b} u^{a}\right)+c_{4}\left(u^{a} \nabla_{a} u^{c}\right)\left(u^{b} \nabla_{b} u_{c}\right)\right) \boldsymbol{\epsilon}
\end{aligned}
$$

where $R$ is the scalar curvature of the metric $g_{a b}$, the $c_{i}$ are constants, and $\epsilon$ is the canonical volume form associated with $g_{a b}$. This Lagrangian is the most general (modulo a boundary term) that is covariant, second-order in derivatives, and consistent with the constraint $u^{a} u_{a}=1$. The constraint can be accounted for by adding to $\mathbf{L}$ a term of the form

$$
\lambda\left(u^{a} u^{b} g_{a b}-1\right) \boldsymbol{\epsilon}
$$

where $\lambda$ is a Lagrange multiplier. 
Varying $\mathbf{L}$, we obtain $\Theta$ :

$$
\begin{aligned}
\Theta_{a b c}=\frac{1}{16 \pi G} \boldsymbol{\epsilon}_{d a b c}\left[g ^ { d e } g ^ { f h } \left(\nabla_{e} \delta g_{f h}\right.\right. & \left.-\nabla_{f} \delta g_{e h}\right) \\
& \left.-\left(2 K_{e}^{d} \delta u^{e}+\left(K^{e f} u^{d}+\left(K^{d f}-K^{f d}\right) u^{e}\right) \delta g_{e f}\right)\right],
\end{aligned}
$$

where

$$
K_{m}^{a}=\left(c_{1} g^{a b} g_{m n}+c_{2} \delta_{m}^{a} \delta_{n}^{b}+c_{3} \delta_{n}^{a} \delta_{m}^{b}+c^{4} u^{a} u^{b} g_{m n}\right) \nabla_{b} u^{n} .
$$

From this follows $\mathbf{J}(3)$ :

$$
\mathbf{J}_{a b c}=\frac{1}{16 \pi G} \boldsymbol{\epsilon}_{\text {dabc }}\left(A^{d e f}{ }_{h} \nabla_{e} \nabla_{f} \xi^{h}+B^{d e}{ }_{h} \nabla_{e} \xi^{h}+C^{d}{ }_{h} \xi^{h}\right),
$$

where

$$
\begin{aligned}
A^{d e f}{ }_{h} & =\left(-g^{e f} \delta_{h}^{d}+g^{d(e} \delta_{h}^{f)}\right), \\
B^{d e}{ }_{h} & =2\left(K^{[d}{ }_{h} u^{e]}+K_{h}{ }^{[d} u^{e]}+K^{[e d]} u_{h}\right),
\end{aligned}
$$

and we will not need the form of $C^{d}{ }_{h}$.

The Noether charge Q (4) can be extracted via an algorithm of Wald [9], yielding

$$
\begin{aligned}
\mathrm{Q}_{a b} & =\frac{1}{16 \pi G} \boldsymbol{\epsilon}_{a b c d}\left[\frac{2}{3} A^{c d f}{ }_{h} \nabla_{f} \xi^{h}+\frac{1}{2} B_{h}^{c d} \xi^{h}\right] \\
& =\frac{1}{16 \pi G} \epsilon_{a b c d}\left[\nabla^{c} \xi^{d}+\left(\left(K_{h}^{c}+K_{h}{ }^{c}\right) u^{d}-K^{c d} u_{h}\right) \xi^{h}\right] .
\end{aligned}
$$

\section{CONSERVED QUANTITIES}

We now consider the expressions for the total energy, momentum, and angular momentum

of an asymptotically flat space-time in Æ-theory. For the requisite integrals to be convergent, we must define fall-off conditions on the fields and their variations. We will assume that at spatial infinity, there exists an asymptotic Cartesian coordinate basis, with respect to which the components of the metric and its derivatives are

$$
g_{\mu \nu}=\eta_{\mu \nu}+O(1 / r)
$$

and

$$
\frac{\partial g_{\mu \nu}}{\partial x^{\alpha}}=O\left(1 / r^{2}\right)
$$


where $\eta_{a b}$ is the flat metric. The variations of the metric $\delta g_{a b}$ must be $O(1 / r)$. For the æther, we require that

$$
u^{\mu}=\bar{u}^{\mu}+O(1 / r),
$$

where asymptotically, $\nabla_{a} \bar{u}^{b}=0$. We can always choose to effect an asymptotic Lorentz boost so that $\bar{u}^{a}=t^{a}$ at infinity, i.e. we are in the rest-frame of the æther. With respect to the asymptotic Cartesian basis,

$$
\frac{\partial u^{\mu}}{\partial x^{\alpha}}=O\left(1 / r^{2}\right) .
$$

The variation $\delta u^{a}$ will be assumed to be $O(1 / r)$.

We turn now to the total energy. One finds in this case that with the above fall-off conditions, $\boldsymbol{\Theta}=\boldsymbol{\Theta}_{G}+O\left(1 / r^{3}\right)$ asymptotically, where $\boldsymbol{\Theta}_{G}$ is the form which arises for GR in vacuum. Hence, we can choose $\mathbf{B}=\mathbf{B}_{G}$, the vacuum form. The total energy can then be written as $\mathcal{E}=\mathcal{E}_{G}+\mathcal{E}_{\mathbb{E}}$, where $\mathcal{E}_{G}$ is the standard ADM mass [8]

$$
\mathcal{E}_{G}=\frac{1}{16 \pi G} \sum_{i, j=1}^{3} \int_{\infty} d S r^{i}\left(\partial_{i} g_{j j}-\partial_{j} g_{i j}\right),
$$

where $d S$ is the spherical area element and $r^{a}=(\partial / \partial r)^{a}$. The æther portion $\mathcal{E}_{\text {E }}$ is

$$
\begin{aligned}
\mathcal{E}_{\text {E }} & =\frac{1}{16 \pi G} \int_{\infty} d S 2 r_{[c} t_{d]}\left(\left(K^{c t}+K^{t c}\right) \bar{u}^{d}-K^{c d} \bar{u}^{t}\right) \\
& =\frac{1}{8 \pi G} \int_{\infty} d S t_{a}\left(r^{b} t_{c}+r_{c} t^{b}\right) K^{a}{ }_{b} \bar{u}^{c} .
\end{aligned}
$$

Setting $\bar{u}^{a}=t^{a}$ gives

$$
\begin{aligned}
\mathcal{E}_{\text {E }} & =\frac{\left(c_{1}+c_{4}\right)}{8 \pi G} \int_{\infty} d S t^{a} r_{b} \nabla_{a} u^{b} \\
& =\frac{\left(c_{1}+c_{4}\right)}{8 \pi G} \int_{\infty} d S\left(\partial_{t} u^{r}+\partial_{r} u^{t}\right),
\end{aligned}
$$

where in the first line we have used the fact that $t_{a} \nabla_{b} u^{a}=u_{a} \nabla_{b} u^{a}+O\left(1 / r^{3}\right)$, and in the second we have used the constraint, which requires $\partial_{\mu} u^{t}=-(1 / 2) \partial_{\mu} g_{t t}+O\left(1 / r^{3}\right)$.

This expression can be evaluated more explicitly for a static, spherically-symmetric, matter-free solution, as the asymptotic forms of the fields are known [12]. In isotropic coordinates, the line element has the form:

$$
d S^{2}=N(r) d t^{2}-B(r)\left(d r^{2}+r^{2} d \Omega\right)
$$


and $\bar{u}^{a}=t^{a}$. Assuming the generic case $c_{1}+c_{2}+c_{3} \neq 0$, one finds that to $O(1 / r), N=$ $1-\left(r_{0} / r\right)$ and $B=1+\left(r_{0} / r\right)$, for arbitrary constant $r_{0}$. The total energy is then

$$
\mathcal{E}=\frac{r_{0}}{2 G}\left(1-\frac{c_{1}+c_{4}}{2}\right)
$$

This result was previously found by Eling using pseudotensor methods [6]. The quantity

$$
G_{N}=G\left(1-\frac{\left(c_{1}+c_{4}\right)}{2}\right)^{-1}
$$

has been identified in studies of the Æ-theory Newtonian limit [3, 13] as the value of Newton's constant that one would measure far from gravitating matter (assuming no direct interaction between æther and non-æther matter). We can define a Newtonian gravitating mass $M=$ $2 r_{0} / G_{N}$, in which case

$$
\mathcal{E}=M
$$

The total momentum in the $x_{i}^{a}$ direction also has the form $\left(\mathcal{P}_{G}\right)_{i}+\left(\mathcal{P}_{\mathbb{E}}\right)_{i}$, where $\left(\mathcal{P}_{G}\right)_{i}$ is the standard ADM momentum [8] ,

$$
\left(\mathcal{P}_{G}\right)_{i}=\frac{1}{16 \pi G} \sum_{j, k=1}^{3} \int d S r^{j}\left(\partial_{0} g_{j i}-\partial_{j} g_{0 i}+\delta_{i j}\left(\partial_{k} g_{0 k}-\partial_{0} g_{k k}\right)\right) .
$$

The æther contribution, setting $\bar{u}^{a}=t^{a}$, is

$$
\left(\mathcal{P}_{\mathbb{E}}\right)_{i}=\frac{-1}{16 \pi G} \int_{\infty} d S\left(c_{1}+c_{3}\right)\left(r^{a} \nabla_{a} u_{i}+r_{a} \nabla_{i} u^{a}\right)+2 c_{2} r_{i} \nabla_{b} u^{b} .
$$

The total angular momentum takes the form $\mathcal{J}_{G}+\mathcal{J}_{\mathbb{E}}$, where $\mathcal{J}_{G}$ is the generalization to a non-axi-symmetric space-time of the conventional Komar expression for vacuum GR [8],

$$
\mathcal{J}_{G}=\frac{1}{16 \pi G} \int_{\infty} d S n_{a b} \nabla^{a} \varphi^{b}
$$

where $n_{a b}$ is the binormal of the boundary of $C$. The æther contribution $\mathcal{J}_{\mathbb{E}}$ is

$$
\mathcal{J}_{Æ}=\frac{-\left(c_{1}+c_{3}\right)}{16 \pi G} \int_{\infty} d S 2 r_{(a} \phi_{b)} \nabla^{a} u^{b}
$$

having set $\bar{u}^{a}=t^{a}$.

\section{FIRST LAW OF BLACK HOLE MECHANICS}

For a stationary black hole space-time, the Noether charge formalism allows one to write down a differential identity that relates variations in the total energy and angular momentum 
to variations of integrals over a cross-section of the horizon. It has been shown [4, 5] that this identity becomes the 'first law' of black hole mechanics/thermodynamics for a wide variety of generally covariant gravitational theories.

A one-parameter family, for a fixed set of $c_{i}$ values, of static, spherically-symmetric ÆEtheory black hole solutions has been shown to exist [7]. The existence proof found in 7] is based on numerical integration of the field equations, and analytic expressions for the fields are only known asymptotically (see 12]). Thus, we cannot obtain a first law by directly examining the solutions. We can instead apply the Noether charge method and attempt to massage the variational identity into a form resembling the familiar first law. The closest we will come here will be for the static, spherically-symmetric case, for which we will show that the identity can be written in the form

$$
\delta M=\frac{\kappa}{8 \pi G}[(1+\phi N) \delta A+\phi A \delta O],
$$

where $M$ is the black hole mass (i.e. the total energy, c.f. (28)), $A$ is the area of the horizon, $N$ and $O$ are quantities depending on the metric, æther, and the local geometry of the horizon, and $\kappa$ and $\phi$ are parameters defined below. Although this expression resembles the familiar first law, it does not lead to an obvious thermodynamic interpretation; in particular, we do not obtain a definitive expression for the horizon entropy.

The variational identity of interest is derived via the Noether charge method by applying Hamilton's equation (11) to perturbations of an asymptotically flat, stationary, axi-symmetric configuration containing a Killing horizon. A Killing horizon is a null hypersurface to which a Killing field is normal - we take it to define the black hole horizon. The Cauchy surface $C$ is assigned a boundary consisting of the 2 -sphere "at infinity" and the surface $B$ where $C$ meets the horizon $\mathcal{H}$. We will assume that $B$ is compact. Choose $\xi^{a}$ to be the horizon-normal Killing field $\chi^{a}$, normalized as

$$
\chi^{a}=t^{a}+\Omega \phi^{a},
$$

where $t^{a}$ is the stationary Killing field with unit norm at infinity, and $\phi^{a}$ is the axi-symmetric Killing field; the constant $\Omega$ defines the angular velocity of the horizon. As $\delta \mathbf{J}[\chi]-d\left(i_{\chi} \boldsymbol{\Theta}\right)$ is linear in $\mathcal{L}_{\chi} \psi=0, \delta H_{\chi}$ vanishes. From the definitions of the total energy (8) and angular momentum (10), the identity emerges:

$$
\delta \mathcal{E}-\Omega \delta \mathcal{J}=\int_{B} \delta \mathbf{Q}-i_{\chi} \mathbf{\Theta} .
$$


Note that the vanishing of $\delta \mathbf{J}[\chi]-d\left(i_{\chi} \boldsymbol{\Theta}\right)$ also implies that the choice of $B$ is arbitrary.

There is no precise definition of a "first-law form" of an expression; roughly speaking, however, by analogy with the conventional thermodynamic expression, a black hole first law should relate variations of "macroscopic" variables - global variables and other parameters that describe the black hole space-time. Considering the explicit form that the identity

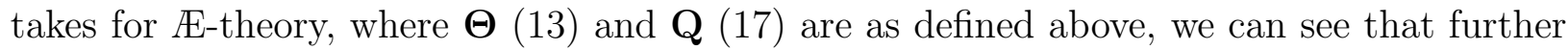
manipulation is required for the identity (35) to take a first-law form.

An algorithm for massaging (35) into such a form and defining the entropy associated with the horizon was given by Wald [4] and improved upon by Iyer and Wald [5]. For the algorithm to apply, it is necessary that the stationary space-time be extendible to one whose Killing horizon possesses a bifurcation surface - a cross-section on which the horizonnormal Killing field vanishes - on which all dynamical fields are regular. In that case, one can work with the extended space-time and choose $B$ to be the bifurcation surface. The algorithm relies on the universal behavior of $\chi^{a}$ in a neighborhood of the bifurcation surface and reduces the horizon terms to the form $(\kappa / 2 \pi G) \delta S$. Here, $\kappa$ is the 'surface gravity' of the horizon, defined by $\kappa^{2}=-\frac{1}{2} \nabla_{a} \chi_{b} \nabla^{a} \chi^{b}$, evaluated on the bifurcation surface, and

$$
S=2 \pi \int_{B} E^{a b c d} n_{a b} n_{c d}
$$

where $n_{a b}$ is the binormal of $B$, and $E^{a b c d}$ is the functional derivative of the Lagrangian with respect to the Riemann tensor $R_{a b c d}$, treating it as a field independent of the metric. General kinematical arguments [14] in the context of quantum field theory in curved spacetime indicate that the temperature due to thermal radiation associated with a Killing horizon is always $\kappa / 2 \pi$. The form of the horizon terms then suggest that we identify $S / G$ as the thermodynamic entropy associated with the horizon.

Unfortunately, the above requirement cannot be met for any Æ-theory configuration [2]. Racz and Wald [15] have shown that a space-time containing a Killing horizon can be extended smoothly to one containing a bifurcation surface if the horizon has compact crosssections and constant, non-vanishing surface gravity. Regular extensions of matter fields on that space-time are not guaranteed. In fact, no such extension can exist for a vector field $u^{a}$ that is invariant under the Killing flow and not tangent to a horizon cross-section. The Killing flow acts at the bifurcation surface as a "radially-directed" Lorentz boost, under which only vectors tangent to the surface can be invariant. In particular, the æther cannot 
possess a regular extension, since it is constrained to be time-like, while a cross-section of a null surface must be space-like.

We can only proceed by less elegant and less general means. We will now restrict attention to the case of a perturbation between spherically-symmetric, static solutions, and show that in this case the variational identity (35) can be written in the form (33).

Consider a variation between static, spherically-symmetric solutions, each containing a Killing horizon $\mathcal{H}$. Identify the solutions such that the horizons coincide, and so that the Killing orbits coincide in a neighborhood of the horizon. That this can be done follows from the construction of "Kruskal-like" coordinates in ref. [15]. We can further define these coordinates so that the variations of the non-angular components of the metric vanish on $\mathcal{H}$ : the line element near $\mathcal{H}$ takes the form

$$
d s^{2}=G d U d V-R^{2} d \Omega^{2}
$$

where $G$ and $R$ are functions of the quantity $U V$, and $\mathcal{H}$ is defined by $U V=0$; we then effect a rescaling of $U$ and $V$ such that $G(0)=1$ for each solution.

Another effect of this identification [16] is that near $\mathcal{H}$, the Killing vector $\chi^{a}$ with surface gravity $\kappa_{0}$ in the unperturbed solution coincides with the Killing vector with the same surface gravity $\kappa_{0}$ in the perturbed solution. From this fact, it follows that on $\mathcal{H}, \delta \mathbf{Q}[\chi]=\kappa \delta \mathbf{Q}[k]$, where $k^{a}=\kappa^{-1} \chi^{a}$ is the unit-surface-gravity Killing field near $\mathcal{H}$ for both configurations, and is held fixed in the variation of $\delta \mathbf{Q}[k]$.

We will consider the portion of $\mathcal{H}$ defined by $U=0, V>0$, and a cross-section $B$ corresponding to some value of $V$. We can define a null dyad on $\mathcal{H}$ consisting of $k^{a}$ and $\bar{k}^{a}$, where $\bar{k}^{a}$ is the unique null vector normal to $B$ such that $k_{a} \bar{k}^{a}=1$. From the vanishing on $\mathcal{H}$ of the variations of $k^{a}$ and the transverse components of the metric, it follows that $\bar{k}^{a}$ is the same vector field for both solutions; i.e. $\delta \bar{k}^{a}=0$. The metric $h_{a b}=-g_{a b}+2 k_{(a} \bar{k}_{b)}$ induced on $B$ has a variation

$$
\delta h_{a b}=\frac{\delta A}{A} h_{a b}
$$

where $A$ is the area of $B$. The æether $u^{a}$ can be decomposed with respect to this dyad:

$$
u^{a}=\frac{1}{2 \phi} k^{a}+\phi \bar{k}^{a} .
$$

where $\phi=u^{a} k_{a}$. We then have

$$
\delta u^{a}=-\frac{\delta \phi}{\phi}\left(\frac{1}{2 \phi} k^{a}-\phi \bar{k}^{a}\right) \equiv-\frac{\delta \phi}{\phi} \bar{u}^{a} .
$$


A non-null dyad normal to $B$ consists of $u^{a}$ and the orthogonal unit-vector $\bar{u}^{a}$. The bi-normal $n_{a b}$ of $B$ can be expressed in various ways:

$$
n_{a b}=2 \bar{k}_{[a} k_{b]}=2 u_{[a} \bar{u}_{b]}=\frac{2}{\phi} k_{[a} \bar{u}_{b]} .
$$

Now, the algorithm cited above can be used to evaluate the æther-independent horizon terms, which give [4, 5] the standard contribution $(\kappa / 8 \pi G) \delta A$. Evaluating the ætherdependent portion of $\mathbf{Q}[k]$, pulled-back to $B$, we find that

$$
\begin{aligned}
\mathbf{Q}_{Æ}[k] & =\frac{1}{16 \pi G} \epsilon n_{a b} k^{c}\left(u^{a}\left(K^{b}{ }_{c}+K_{c}{ }^{b}\right)+u_{c} K^{a b}\right) \\
& =\frac{1}{8 \pi G} \boldsymbol{\epsilon} k_{a} \bar{u}_{b} K^{a b} \\
& =\frac{1}{8 \pi G} \boldsymbol{\epsilon} \phi\left(c_{14} n^{a}{ }_{b}-c_{123} \delta_{b}^{a}-c_{13} h_{b}^{a}\right) \nabla_{a} u^{b},
\end{aligned}
$$

where $\boldsymbol{\epsilon}$ is the volume-element of $B, h_{b}^{a}=g^{a c} h_{c b}$, and we have written, e.g. $c_{14}$ for $c_{1}+c_{4}$.

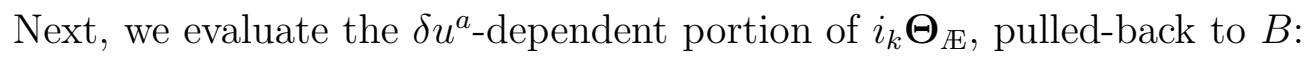

$$
\frac{1}{8 \pi G} \boldsymbol{\epsilon} n_{a b} k^{b} K_{c}^{a} \delta u^{c}=\frac{1}{8 \pi G} \boldsymbol{\epsilon} \frac{\delta \phi}{\phi} k_{a} \bar{u}_{b} K^{a b}=\frac{\delta \phi}{\phi} \mathbf{Q}_{\mathbb{E}}[k] .
$$

The portion containing metric variations gives

$$
\frac{1}{16 \pi G} \boldsymbol{\epsilon} n_{a b} k^{a} u^{b} \delta h_{c d} K^{c d}=\frac{\phi}{16 \pi G} \epsilon \frac{\delta A}{A}\left(c_{13} h_{b}^{a}-2 c_{2} \delta_{b}^{a}\right) \nabla_{a} u^{b} .
$$

We thus have

$$
\begin{aligned}
\left.\int_{B}\left(\delta \mathbf{Q}_{[} \chi\right]-i_{\chi} \boldsymbol{\Theta}\right)=\frac{\kappa}{8 \pi G}\left[\left(1+\phi\left(c_{14} n_{b}^{a}-\right.\right.\right. & \left.\left.c_{13}\left(\delta_{b}^{a}+\frac{3}{2} h_{b}^{a}\right)\right) \nabla_{a} u^{b}\right) \delta A \\
& \left.+\phi A \delta\left(\left(c_{14} n_{b}^{a}-c_{123} \delta_{b}^{a}-c_{13} h_{b}^{a}\right) \nabla_{a} u^{b}\right)\right],
\end{aligned}
$$

and we can then write the variational identity (35) in the form (33).

Although we have obtained this first-law form, a thermodynamic interpretation of it has not emerged. In particular, we do not have a definitive expression for the horizon entropy. For variations between members of a one-parameter family of solutions, the horizon terms (45) must be reducible to $(\alpha \kappa / 2 \pi G) \delta A$ for some dimensionless constant $\alpha$. Even with the Noether charge approach, however, we still cannot discern the value of $\alpha$, nor do we know whether $\alpha A / G$ acts as the entropy in the non-static case.

It is possible that this confusion is related to an obscurity in the notion of a black hole horizon in Æ-theory. Linearized perturbations about a 'flat' background (flat space-time 
and constant-æther) were investigated in [17]. It was found that there exist five independent wave-modes that travel at three different $c_{i}$-dependent speeds. These speeds generally differ from the "speed of light" defined by the flat metric, and exceed it for certain $c_{i}$ values. The behavior of perturbations about a curved background is not known, but we can conjecture that a similar result holds. If that is so, then a Killing horizon is not generally a causal horizon. On the other hand, the perturbations about the flat background do all propagate on the light-cones of the flat metric [17] in the special case $c_{13}=c_{4}=0, c_{2}=c_{1} /\left(1-2 c_{1}\right)$, but the expression (45) does not drastically simplify in this case.

Given the wide applicability of the principles of black hole thermodynamics in generally covariant theories of gravity, it would be surprising if they did not apply to Æ-theory. It seems likely that the problem of formulating the laws of Æ-theory black hole mechanics has a simple solution, which would become apparent if we knew more about the explicit form of the solutions in the neighborhood of the horizon.

\section{Acknowledgments}

I thank Robert Wald and Ted Jacobson for discussions, and the Institut d'Astrophysique de Paris for hospitality while this work was begun. This research was supported in part by the NSF under grant PHY-0300710 at the University of Maryland.

[1] J. W. Elliott, G. D. Moore and H. Stoica, JHEP 0508, 066 (2005).

[2] C. Eling, T. Jacobson and D. Mattingly, arXiv:gr-qc/0410001.

[3] B. Z. Foster and T. Jacobson, arXiv:gr-qc/0509083.

[4] R. M. Wald, Phys. Rev. D 48, R3427 (1993).

[5] V. Iyer and R. M. Wald, Phys. Rev. D 50, 846 (1994).

[6] C. Eling, arXiv:gr-qc/0507059.

[7] C. Eling and T. Jacobson, unpublished.

[8] R. M. Wald, General Relativity, (University of Chicago Press, 1984).

[9] R. M. Wald, J. Math. Phys. 31, 2378 (1990).

[10] J. M. Cohen and R. M. Wald, J. Math. Phys. 13, 543 (1972). 
[11] S. Yoshida and Y. Eriguchi, Phys. Rev. D 56, 762 (1997); F. E. Schunck and E. W. Mielke, Class. Quant. Grav. 20, R301 (2003).

[12] C. Eling and T. Jacobson, Phys. Rev. D 69, 064005 (2004).

[13] S. M. Carroll and E. A. Lim, Phys. Rev. D 70, 123525 (2004);

[14] B. S. Kay and R. M. Wald, Phys. Rept. 207, 49 (1991).

[15] I. Racz and R. M. Wald, Class. Quant. Grav. 13, 539 (1996).

[16] T. Jacobson, G. Kang and R. C. Myers, Phys. Rev. D 49, 6587 (1994).

[17] T. Jacobson and D. Mattingly, Phys. Rev. D 70, 024003 (2004). 